\title{
FLAVONÓIDES E TRITERPENOS DE Baccharis pseudotenuifolia - BIOATIVIDADE SOBRE Artemia salina
}

Francisco de Paula Madeira Moreira, Valmor Coutinho, Ana Beatriz Pimentel Montanher, Miguel Soriano Balparda Caro, Inês Maria Costa Brighente* e Moacir Geraldo Pizzolatti

Departamento de Química, Universidade Federal de Santa Catarina, CP 476, 88040-900 Florianópolis - SC

Franco Delle Monache

Centro Chimica Recettori, Consiglio Nazionale delle Ricerche, Largo Francesco Vito 1, 00168 Roma-Italy

Recebido em 14/3/02; aceito em 5/12/02

FLAVONOIDS AND TRITERPENES FROM Baccharis pseudotenuifolia - BIOACTIVITY ON Artemia salina. The phytochemical investigation of the chloroformic and methanolic extracts of the Baccharis pseudotenuifolia led to the isolation of triterpenes, steroids and flavonoids. From chloroformic extract were isolated oleanolic acid and $\alpha$-spinasterol while from methanolic extract were isolated the flavonoids: hispidulin, naringenin, 3'-methoxy-luteolin, apigenin, kaempferol, eriodyctiol, aromadendrin, quercetin, 3'-methoxy-quercetin, quercetin-3-O-rhamnoside and quercetin-3-O-glucoside. The structure of these compounds were identified by IR, CG/MS, ${ }^{1} \mathrm{H}$ and ${ }^{13} \mathrm{C}$ NMR spectral analysis and comparison with literature. In addition, the extracts were evaluated by means of Brine Shrimp Lethality test and the highier activity was observed in the chloroformic extract.

Keywords: Baccharis pseudotenuifolia; Artemia salina; flavonoids.

\section{INTRODUÇÃO}

Plantas do gênero Baccharis, conhecidas popularmente por carquejas, são arbustos lenhosos de grande diversidade morfológica pertencentes à família Asteraceae, uma das maiores famílias que florescem (angiospermas), onde suas 25.000 espécies, distribuídas no mundo todo, estão divididas em 1.100 gêneros ${ }^{1}$.

O gênero Baccharis está representado por mais de 500 espécies nativas $^{2}$ e exclusivas das Américas que estão distribuídas principalmente no Brasil e montanhas andinas ${ }^{3}$. No Brasil são aproximadamente cerca de 120 espécies, segundo Barroso ${ }^{4}$. Muitas espécies são utilizadas na medicina folclórica para o tratamento de males do estômago $^{5}$, desordens hepáticas e renais ${ }^{6}$, com atividades antiinflamatória $^{7}$, antidiabética ${ }^{8}$ e abortiva $^{9}$ e na produção de fragrâncias comerciais (óleo de carqueja) ${ }^{10}$. Algumas espécies são reconhecidamente tóxicas apesar de seu uso na medicina popular. Nos países da América do Sul, a morte de bovinos e ovinos ao se alimentarem com folhas de Baccharis ${ }^{11-13}$ levou à avaliação da atividade citotóxica ${ }^{14-18}$ destas espécies.

Do ponto de vista fitoquímico, apesar de não mais que $15 \%$ das espécies terem sido estudadas, quanto à sua composição micromolecular, pode-se observar que o gênero é caracterizado pelo acúmulo de sesquiterpenos, diterpenos, triterpenos e flavonóides ${ }^{19-23}$.

No presente trabalho realizamos a investigação fitoquímica de B. pseudotenuifolia, juntamente com a avaliação do efeito citotóxico dos extratos, utilizando ensaios com A. salina $^{24}$.

\section{PARTE EXPERIMENTAL}

\section{Material vegetal}

Folhas de Baccharis pseudotenuifolia foram coletadas nos arredores de Porto Alegre. Uma exsicata foi depositada no Departamento de Botânica do Instituto de Biociência da UFRGS sob o $\mathrm{N}^{\circ} \mathrm{ICN}$ 535773.

* e-mail: ines@qmc.ufsc.br

\section{Isolamento dos triterpenos e esteróis}

O extrato em clorofórmio da espécie $B$. pseudotenuifolia foi submetido a um pré-fracionamento por filtração em leito de $\mathrm{CaCO}_{3}-$ $\mathrm{Na}_{2} \mathrm{CO}_{3}$-Celite, utilizando-se como eluentes clorofórmio e metanol. Desta forma obteve-se duas frações: uma fração lipofílica eluída com clorofórmio, constituída essencialmente de ceras e uma fração metanólica. A fração metanólica foi submetida ao fracionamento por cromatografia em coluna de sílica gel, utilizando-se como eluente hexano/acetato de etila em gradiente de polaridade. Com este procedimento obteve-se o isolamento do ácido oleanólico $(\mathbf{1})^{25}$. O fracionamento da fração lipofílica em coluna de sílica, eluída com gradiente hexano/acetato de etila, resultou no isolamento de $\alpha$ espinasterol $(\mathbf{2})^{26,27}$.

\section{Isolamento dos flavonóides}

O extrato metanólico de $B$. pseudotenuifolia foi cromatografado em coluna de sílica gel eluída com mistura hexano/acetato de etila/ metanol no modo gradiente de polaridade. As frações que deram reação positiva com o regente $\mathrm{FeCl}_{3}$ em cromatografia em camada delgada (ccd) foram reunidas em dois grupos. O grupo de menor polaridade foi recromatografado em coluna de sílica gel (gradiente: hexano-acetato de etila) e as frações contendo flavonóides (ccd- $\mathrm{FeCl}_{3}$ ) foram submetidas a sucessivas cromatografias em coluna de sílica flash ${ }^{28}$ sob pressão e purificações por recristalizações, resultando no isolamento dos flavonóides $\mathbf{3}$ a 10. O grupo mais polar foi submetido a uma gel filtração em Sephadex LH-20 resultando em duas frações flavonoídicas, segundo ccd- $\mathrm{FeCl}_{3}$. A recromatografia destas frações em coluna de sílica eluída com clorofórmio/metanol no modo gradiente de polaridade resultou no isolamento de quercetina $\mathbf{1 1}$ e na obtenção da mistura quercetina-3-O-ramnosídeo 12 e quercetina-3O-glicosídeo 13.

\section{Bioensaio com Artemia salina}

Utilizou-se a metodologia de Meyer $^{29}$ adaptada. Cerca de 10 lar- 
<smiles>[R2]c1cc([C@H]2Oc3cc(O)cc(O)c3C(=O)C2[R])ccc1O</smiles><smiles>[R]c1cc(-c2oc3cc(O)c([R])c(O)c3c(=O)c2[R])ccc1O</smiles>

$\begin{array}{rll} & \mathrm{R}_{1} & \mathrm{R}_{2} \\ 4 & \mathrm{H} & \mathrm{H} \\ 9 & \mathrm{H} & \mathrm{OH} \\ 10 & \mathrm{OH} & \mathrm{H}\end{array}$

$\begin{array}{rlll} & \mathrm{R}_{1} & \mathrm{R}_{2} & \mathrm{R}_{3} \\ & & & \\ 3 & \mathrm{H} & \mathrm{H} & \mathrm{OMe} \\ \mathbf{5} & \mathrm{H} & \mathrm{OMe} & \mathrm{H} \\ \mathbf{6} & \mathrm{H} & \mathrm{H} & \mathrm{H} \\ 7 & \mathrm{OH} & \mathrm{H} & \mathrm{H} \\ 8 & \mathrm{OH} & \mathrm{OMe} & \mathrm{H} \\ 11 & \mathrm{OH} & \mathrm{OH} & \mathrm{H} \\ 12 & \mathrm{O}-\mathrm{Ram} & \mathrm{OH} & \mathrm{H} \\ 13 & \text { O-Gluc } & \mathrm{OH} & \mathrm{H}\end{array}$

vas de Artemia salina foram transferidas para tubos de ensaios contendo água artificial do mar e a substância a ser testada, em quatro diferentes concentrações. Os testes foram feitos em triplicata. A contagem dos animais mortos e vivos foi realizada após $24 \mathrm{~h}$. Utilizouse o método Probitos de análise para obtenção das $\mathrm{DL}_{50}$ e respectivos intervalos de confiança ${ }^{30}$. Os extratos foram considerados ativos quando $\mathrm{DL}_{50}<1000 \mathrm{ppm}^{29}$. As substâncias puras testadas foram o ácido oleanólico ( $\left.\mathrm{DL}_{50} 85 \mathrm{ppm}\right)$ e a hispidulina ( $\left.\mathrm{DL}_{50} 468 \mathrm{ppm}\right)$. Cada teste foi acompanhado de um controle negativo e positivo com $\mathrm{K}_{2} \mathrm{Cr}_{2} \mathrm{O}_{7}\left(\mathrm{DL}_{50}=20 \mathrm{a} 40 \mathrm{ppm}\right)^{31}$.

\section{RESULTADOS E DISCUSSÃO}

Os extratos em clorofórmio e em metanol de folhas da espécie Baccharis pseudotenuifolia foram submetidos ao bioensaio de toxicidade sobre Artemia salina (TAS), sendo que ambos foram considerados ativos (TAS $<1000 \mathrm{ppm}^{29}$, Tabela 1). A maior atividade concentrou-se no extrato clorofórmico (TAS $=115 \mathrm{ppm}$ ), no qual detectou-se a presença do triterpeno ácido oleanólico (1), através de ccd revelado com o reagente de Liebermann-Burchard. Este resultado é indicativo da presença de um importante componente citotóxico na fração clorofórmica. Desta fração foram isolados $\alpha$-espinasterol e ácido oleanólico, identificados por comparação direta em ccd e pela comparação dos dados de RMN ${ }^{13} \mathrm{C}$ com padrões descritos na literatura ${ }^{25-27}$. A realização do bioensaio com os compostos isolados demonstrou que o ácido oleanólico (TAS $=85 \mathrm{ppm}$ ) pode ser o princípio ativo responsável pela atividade do extrato clorofórmico nesta espécie de Baccharis. Kubo e Matsumoto reportaram ${ }^{32}$ que o ácido oleanólico encontrado nas folhas de $B$. linearis pode constituir uma barreira física na prevenção de ataques contra microorganismos. No extrato metanólico encontrou-se a hispidulina como flavonóide majoritário, a qual pode ser responsável pela atividade neste extrato (Tabela 1).

Tabela 1. Teste de toxidade para A. salina dos extratos e compostos isolados de Baccharis pseudotenuifolia

\begin{tabular}{lcc}
\hline & $\mathrm{DL}_{50}(\mathrm{ppm})$ & $\begin{array}{c}\text { Intervalo de confiança } \\
95 \%(\mathrm{ppm})\end{array}$ \\
\hline Extrato clorofórmico & 115 & $83-157$ \\
Extrato metanólico & 813 & $676-983$ \\
Ácido oleanólico & 85 & $69-105$ \\
Hispidulina & 468 & $389-563$ \\
$\mathrm{~K}_{2} \mathrm{Cr}_{2} \mathrm{O}_{7}$ & $20-40^{*}$ & \\
\hline
\end{tabular}

*Ref. 31
O fracionamento cromatográfico do extrato metanólico das folhas de B. pseudotenuifolia resultou no isolamento das flavanonas 4 e 9, do flavanonol 10, das flavonas 3, 5 e 6, dos flavonóis 7, 8 e 11 e na separação de uma mistura contendo os glicosídeos quercetina-3O-ramnosídeo 12 e quercetina-3-O-glucosídeo 13.

Os espectros de absorção na região do IV dos compostos 3-11 apresentaram um perfil de absorção típico de flavonóides, com intensa absorção de $\mathrm{OH}$ na região de 3280 a $3410 \mathrm{~cm}^{-1}$, carbonila conjugada envolvida em ligação de hidrogênio intramolecular (1640$1670 \mathrm{~cm}^{-1}$ ) e um grupo de absorções na região de aromáticos, enquanto que os compostos $\mathbf{1 2}$ e $\mathbf{1 3}$ apresentam uma forte e larga banda na região de $3400 \mathrm{~cm}^{-1}$, sugerindo uma estrutura glicosídica. Os espectros de $\mathrm{RMN}{ }^{1} \mathrm{H}$ dos compostos 4-13 apresentaram sinais para um $\mathrm{OH}$ quelado e um par de dupletes na região de hidrogênios aromáticos com constantes de acoplamento para hidrogênios meta correlacionados $(J \sim 2 \mathrm{~Hz})$, típico de flavonóides com anel "A" substituídos nas posições 5 e 7, sugerindo que estes flavonóides são 5,7diidroxi.

Os espectros de $\mathrm{RMN}{ }^{1} \mathrm{H}$ das flavanonas 4 e 9 mostram o sistema $\mathrm{ABX}$ na região de hidrogênios alifáticos $(4: \delta 5,36 \mathrm{dd}, \mathrm{H}-2 ; 2,53 \mathrm{dd}$ H-3a; 3,10 dd H-3b e 9: $\delta$ 5,39 dd H-2; 2,40 dd H-3a; 3, 14 dd H-3b) e os espectros de $\mathrm{RMN}{ }^{13} \mathrm{C}$ exibem dois grupos oxi metínicos (4: $\delta$ 79,35 C-2 e 43,33 C-3 e 9: 79,88 C-2 e 43,46 C-3), caracterizando o esqueleto flavanona. Assim, foram identificados naringenina (4) e eriodictiol (9), cujos dados espectroscópicos concordam com os valores da literatura ${ }^{33}$. O flavanonol 10 é aromadendrina e a flavona 6 é apigenina. Ambos identificados por comparação dos dados de RMN ${ }^{1} \mathrm{He} \mathrm{e}^{13} \mathrm{C}$ com os valores da literatura ${ }^{33}$ e por cromatografia em camada delgada em comparação com amostra autêntica.

Os compostos $\mathbf{3}, \mathbf{5}$ e $\mathbf{6}$ foram identificados como flavonas a partir da observação nos espectros de $\mathrm{RMN}{ }^{1} \mathrm{H}$ de singletos em $\delta 6,95$, 6,98 e 6,64 atribuídos aos H-3, respectivamente. A análise do espectro de RMN ${ }^{1} \mathrm{H}$ do composto $3\left(\mathrm{M}^{+} \cdot 300\right)$ mostrou a presença de sinais para uma metoxila $(\delta 3,99)$ e dois singletos em $\delta 6,93$ e 6,95 , além do par de dupletes típico de anel "B" p-dissubstituído. O espectro de RMN bidimensional de correlação heteronuclear HETCOR $-{ }^{1} J_{\mathrm{H}-\mathrm{C}}$ mostrou que o singleto em $\delta 6,95(\mathrm{H}-3)$ correlaciona com o carbono em $\delta 103,19$ e o singleto em $\delta 6,93(\mathrm{H}-8)$ com o carbono em $\delta 94,97$. A posição da metoxila em C-6 foi deduzida com base na comparação dos deslocamentos químicos do carbono $\delta 94,1$ com 5,7,4'-triidroxi 8-metoxiflavona ( $\delta 99,1$ para C-6) e 5,7,4'-triidroxi 6-metoxiflavona ( $\delta 94,1$ para $C-8)^{31}$. Assim, o composto 3 foi identificado como sendo 5,7,4'-triidroxi-6-metoxi flavona e seus dados de carbono-13 conferem com os da literatura ${ }^{33,34}$.

A posição da metoxila em C-3' no composto 5 foi deduzida através dos experimentos de NOE diferencial. Irradiando a metoxila $\delta$ 3,84 observou-se ressonância em $\delta 7,67$ (H-2'), e a irradiação em $\delta$ 7,63 mostrou ressonância em $\delta 3,84$ e $\delta$ 6,98 (H-3) permitindo a identificação de 5 como sendo 5,7,4'-triidroxi-3'-metoxiflavona.

Os compostos $\mathbf{7}$ e $\mathbf{1 1}$ foram primeiramente identificados como kaenferol e quercetina, respectivamente, através da comparação direta com amostras autênticas em ccd. Os deslocamentos químicos dos átomos de hidrogênio e carbono nos espectros de RMN ${ }^{1} \mathrm{H}$ e RMN ${ }^{13} \mathrm{C}$ estão consistentes com os valores da literatura ${ }^{33,35}$.

$\mathrm{O}$ composto $8\left(\mathrm{M}^{+} \cdot 316\right)$ apresentou um espectro de RMN ${ }^{1} \mathrm{H}$ semelhante ao da quercetina com um sinal adicional para metoxila $(\delta 3,98)$. Experimentos de NOE diferencial (irradiação da metoxila $\delta 3,98$ destaca o sinal em $\delta 8,30$ H-2'; irradiação em $\delta 8,30$ destaca o sinal em $\delta 3,98$ ) permitiram localizar a metoxila em C-3' e a identificação do composto 8 como 3,5,7,4'-tetrahidroxi-3'-metoxiflavona ${ }^{33}$.

Os glicosídeos quercetina-3-O-glucosídeo 12 e quercetina-3-Oramnosídeo $\mathbf{1 3}$ foram identificados em mistura, através de minuciosa análise dos espectros de $\mathrm{RMN}{ }^{1} \mathrm{H}$ e ${ }^{13} \mathrm{C}$ e por comparação com os 
dados da literatura ${ }^{33}$. Na região de açúcares do espectro de $\mathrm{RMN}{ }^{1} \mathrm{H}$ pode-se identificar a presença de dois monossacarídeos através de seus hidrogênios anoméricos em $\delta 5,22$ e $\delta 5,51$, que estão correlacionados no espectro HETCOR aos carbonos anoméricos $\delta 102,5$ e $\delta 105,6$, respectivamente. A presença de uma metila em $\delta 0,91(\mathrm{~d}$, $J=5,59 \mathrm{~Hz}$ ) no espectro de $\mathrm{RMN}{ }^{1} \mathrm{H}$ e $\delta 17,70$ no espectro de RMN ${ }^{13} \mathrm{C}$ sugere a presença de uma unidade de ramnose, que é confirmada pelos valores dos deslocamentos químicos dos carbonos no espectro de $\mathrm{RMN}{ }^{13} \mathrm{C}$ em comparação com a literatura ${ }^{33,36}$. Já a unidade de glucose foi inferida com base nos dados de $\mathrm{RMN}{ }^{13} \mathrm{C}$ em comparação com os valores encontrados na literatura ${ }^{33,36}$. A posição das unidades glicosídicas na posição C-3 foi deduzida pelas diferenças nos deslocamentos químicos dos carbonos C-2 e C-3 do glicosídeo em comparação com os da aglicona quercetina.

\section{REFERÊNCIAS}

1. Jeffery, C. Em Flowering Plants of the World; Bateman, G.; Dodd, B.; Lenthal, B., eds.; Oxford University Press: London, 1970, p. 263.

2. Nesom, G. L.; Phytologia 1990, 69, 40.

3. Herz, W. Em The Biology and Chemistry of Compositae; Heywood, V. H.; Harbone, J. B.; Turner, B. L., eds.; Academic Press: New York, 1977, p. 567.

4. Barroso, G.; Rev. Rodriguesia 1976, 40, 7.

5. Jaime, G.; Arias, M.; Neukman, L.; Anales de SAIPA 1994, 12, 225.

6. Hischmann, G.; De Arias, A. R.; J. Ethnopharmacol. 1990, $29,159$.

7. Bandoni, A. L.; Mendiondo, M. E.; Rondina, R. V. D.; Coussio, J. D.; Lloydia 1972, 35, 69.

8. Korbes, Ir. C. V; Manual de Plantas Medicinais, Equipe Técnica da Assesoar Ed., 44ª ed., Paraná, 1993.

9. Giberti, G. C.; J. Ethnopharmacol.1983, 7, 321

10. Mortl, A.; Trka, A.; Parfum Kosmet 1983, 64, 488.

11. Andriew, A.; Rev. Centro de Estudientes de Agron. 1907, 3, 118.

12. Schang, R.; Rev. Med. Buenos Aires 1929, 11, 151.
13. Torkania, C. E.; Dobereiner, J.; Pesq. Agropec. Brasil. Ser. Vet. 1975, 10, 79.

14. Mongelli, E.; Desmarchelier, C.; Rodriguez Talou, J.; Coussio, J.; Ciccia, G.; J. Ethnopharmacol. 1997, 58, 157.

15. Faini, F.; Labbé, C.; Coll, J.; Biochem. Syst. Ecol. 1999, 27, 673.

16. Fullas, F.; Hussain, R. A.; Chai H.-B.; Pezzuto, J. M.; Soejarto, D. D.; Kinghorn, A. D.; J. Nat. Prod. 1994, 57, 801.

17. Misawa, M.; Hayashi, M.; Takayama, S.; Planta Med. 1983, 2, 115.

18. Bhakuni, D. S.; Bittner, M.; Marticorena, C.; Silva, M.; Weldt, E.; Hoeneisen, M; Hartwell, J. L.; I. Lloydia 1976, 39, 225.

19. Jarvis, B. B.; Mokhtari-Rejali, N.; Schenkel, E. P.; Barros, C. S.; Matzenbacher, N. I.; Phytochemistry 1991, 30, 789.

20. Arriaga, F. J.; Wollenweber, E.; Schober, I.; Dostal, P.; Braun, S.; Phytochemistry 1986, 25, 719.

21. Zdero, C.; Bohlmann, F.; Niemeyer, H. M.; Phytochemistry 1991, 30, 1597.

22. Rivera, A. P.; Arancibia, L.; Casillo, M.; J. Nat. Prod. 1989, 52, 433.

23. Faini, F. A.; Castillo, M.; Torres, M. R.; J. Nat. Prod. 1982, 45, 501.

24. McLaughlin, J. L. Em Methods in Plant Biochemistry; Hostettmann, K., ed.; Academic Press: London, 1991, vol. 6, p. 1

25. Mohato, S. B.; Kundu, A. P.; Phytochemistry 1994, 37, 1517.

26. Garg, V. K.; Nes, W. R.; Phytochemistry 1984, 23, 2919.

27. Garg, V. K.; Nes, W. R.; Phytochemistry 1984, 23, 2925.

28. Still, W. C.; Kahan, M.; Mitra, A.; J. Org. Chem. 1978, 43, 2923.

29. Meyer, B. N.; Ferrigini, N. R.; Putnan, J. E.; Jacobsen, L. B.; Nichols, D. E.; McLaughlin, J. L.; Planta Med. 1982, 45, 31.

30. Finney, D. L. Em Probit Analysis; $3^{\circ}$ ed., Cambridge University Press: Cambridge, 1971, p. 76-80.

31. Sam, T. W. Em Bioactive Natural Products; Colegata, S. M.; Molyneuse, R. J., eds.; CRC Press, Inc.: USA, 1993, cap. 18, p. 450.

32. Kubo, I.; Matsumoto, A.; Experientia 1984, 40, 937.

33. Agrawal, P. K.; Bansal, M. C. Em Carbon-13 NMR of Flavonoids; Agrawal, P. K., ed.; Elsevier: New York, 1989, p. 103, 135 e 155.

34. Barrero, A. F.; Sanchez, J. F.; Rodriguez, I.; Soria Sanz, C.; Anales de Quimica 1984, 84, 344.

35. Oliveira, M. C. C. de; Carvalho, M. G.; Ferreira, D. T.; Braz-Filho, R.; Quim. Nova 1999, 22, 182.

36. Agrawal, P. K.; Phytochemistry 1992, 31, 3307. 